BULL. AUSTRAL. MATH. SOC.

$16 A 12,06 A 70$

VOL. $12(1975), 311-314$.

(16A34, 06A40, 06A55)

\title{
The hulls of semiprime rings
}

\section{Paul F. Conrad}

Each semiprime ring admits a unique projectable, strongly projectable, laterally complete and orthocomplete hull. Almost all of the theory for $X$-hulls of lattice-ordered groups in Paul Conrad, "The hulls of representable $Z$-groups and f-rings", J. Austral. Math. Soc. 16 (1973), 385-415, has a counterpart for semiprime rings. The proofs of these results will appear elsewhere. They come in a large part directly from the corresponding theory for lattice-ordered groups. There is also a feedback from the rings to the groups.

Let $G$ be a semiprime ring and for $a, b \in G$ define $a \geq b$ if $a g b=b g b$ for all $g \in G$. This is equivalent ot the fact that $a$ agrees with $b$ on the support of $b$ in each representation of $G$ as a subdirect product of prime rings. Thus $\geq$ is a partial order for $G$ with smallest element 0 and for $a, b, x \in G$,

$$
a \geq b \text { implies } a x \geq b x, \quad x a \geq x b \text { and } a b=b a .
$$

We say that $a$ is disjoint from $b$ or that $a$ is orthogonal to $b$ if $a G b=0$ (notation $a \perp b$ ). This is equivalent to the fact that $a$ and $b$ have disjoint support in each representation of $G$ as a subdirect product of prime rings. Thus $a \perp b$ if and only if $b \perp a$, and in this case $a b=b a$. If $X$ is a subset of $G$ then

$$
X^{\prime}=\{g \in G \mid g \perp x \text { for each } x \in X\}
$$

is the annihilator ideal of $\boldsymbol{X}$. Lambek [5] has shown that these ideals form a complete boolean algebra which we will denote by $P(G) . G$ will be called

Received 23 December 1974. 
a P-ring if $G=g^{\prime \prime} \oplus g^{\prime}$ for each $g \in G$ (projectable), an SP-ring if $G=X^{\prime \prime} \oplus X^{\prime}$ for each subset $X$ of $G$ (strongly projectable),

an L-ring if each pairwise disjoint subset of $G$ has a least upper bound (laterally complete).

an $O-r i n g$ if $G$ is both an $L$-ring and an SP-ring (orthocomplete).

An overring $H$ is a left essential extension of $G$ if this is the case when $H$ is considered as a left $G$-module. We prove the following theorems for $X=P, S P, L$ or $O$.

THEOREM A. Let $G$ be a semiprime ring and let $H$ be a left essential extension of $G$ that is an $X-r i n g$. Then the intersection of all the subrings of $H$ that contain $G$ and are X-rings is a minimal left essential extension of $G$ that is on $X-r i n g$; called an $X-h u l l$ of $G$.

THEOREM B. Each semiprime ring admits a unique X-hulZ $G^{X} \cdot G^{X}$ is semiprime and $G^{X}$ is reduced (commutative) if and only if $G$ is reduced (commutative). If $G$ has an identity 1 , then 1 is also the identity for $G^{X}$. Finally $G^{X}$ is the minimal right essential extension of $G$ that is an $X$-ring.

If $G$ is reduced then the proofs of these theorems are almost identical with the proofs of the corresponding theorems for lattice-ordered groups in [3]; one simply replaces $a \wedge b$ by $a b$. For semiprime rings the proofs in [3] can be adapted. We show that

$$
G \subseteq G^{P} \subseteq G^{S P} \subseteq\left(G^{S P}\right)^{L}=\left(G^{P}\right)^{L}=G^{0}
$$

and $\left(G^{L}\right)^{P}=\left(G^{L}\right)^{S P} \subseteq G^{0}$, but here we need not have equality.

In order to prove Theorems $A$ and $B$ we show that if $H$ is a left essential extension of the semiprime ring $G$ then $H$ is semiprime and there is a natural isomorphism of $P(H)$ onto $P(G)$. If $H$ is laterally complete then $G$ is an L-subring of $H$ (that is, for each disjoint subset $\left\{g_{\lambda} \mid \lambda \in \Lambda\right\}$ of $G$ for which $v_{G} g_{\lambda}$ exists, it follows that $\left.v_{H} g_{\lambda}=v_{G} g_{\lambda}\right)$. 
If $G$ is a boolean ring then so is $G^{X}$ and $G^{L}=G^{O}$. Moreover $G^{0}$ is the Dedekind-MacNeille completion of $G$ if and only if $G$ has an identity. If $G$ is regular then so is $G^{P}, G^{S P}$, and $G^{0}$. We show that the ring $G^{X}$ is determined by the addition and the partial order.

THEOREM. Suppose that $G$ is a semiprime ring cond consider the system $\left(G^{X},+, \geq\right)$. Then there is a unique multiplication on $G^{X}$ so that

(a) $G^{X}$ is a semiprime ring,

(b) $G$ is a subring of $G^{X}$, and

(c) the multiplication on $G^{X}$ induces the given partial order $\geq$.

Almost all of the theory for the $X$-hulls of lattice-ordered groups in [3] has a counterpart for semiprime rings. In particular, this is true for the annihilator preserving endomorphisms of $G$ and for the theory of semiprime rings with a basis.

$P(G)$ is atomic if and only if $G^{0}$ is a product of prime rings. From this it is easy to derive necessary and sufficient conditions for a reduced ring to be a product of integral domains; in particular, those in the literature for commutative rings (see, for example, [7], Theorem 4.3).

Abian [1] proved that a commutative semiprime ring $G$ is a product of fields if and only if $G$ is hyperatomic and laterally complete. A student of mine, Otis Kenny, has shown that a reduced ring $H$ is a product of division rings if and only if $H$ is hyperatomic and laterally complete. Thus $H^{L}$ is a product of division rings if and only if $H^{\prime}$ is hyperatomic.

If $G$ is a commutative semiprime ring with 1 , then $G^{p}$ is the Baer extension of $G$ that was introduced by Kist [4] and $G^{S P}$ is the Baer extension of $G$ that was introduced by Mewborn [6]. Thus for an arbitrary semiprime ring $G$ with $I$ we have the unique Baer hulls $G$ and $G^{S P}$.

In [8] Speed,using the technique developed in [2] (which is somewhat 
cruder than that used in [3]), constructed $G^{P}$ and $G^{S P}$ and some hulls in between for commutative semiprime rings with 1 . His description of these hulls is categorical, but somewhat complicated.

If $G$ is a semiprime ring then the complete ring of left (right) quotients of $G$ is an 0 -ring that contains $G^{0}$.

\section{References}

[1] Alexander Abian, "Direct product decomposition of commutative semisimple rings", Proc. Amer. Math. Soc. 24 (1970), 502-507.

[2] Paul Conrad, "The lateral completion of a lattice-ordered group", Proc. London Math. Soc. (3) 19 (1969), 444-486.

[3] Paul Conrad, "The hulls of representable 2 -groups and f-rings", $J$. Austral. Math. Soc. 16 (1973), 385-415.

[4] Joseph Kist, "Minimal prime ideals in commutative semigroups", Proc. London Math. Soc. (3) 13 (1963), 31-50.

[5] Joachim Lambek, "On the structure of semi-prime rings and their rings of quotients", Canad. J. Math. 13 (1961), 392-417.

[6] Angel C. Mewborn, "Regular rings and Baer rings", Math. Z. 121 (1971), $211-219$.

[7] T.P. Speed, "A note on commutative Baer rings", J. Austral. Math. Soc. 14 (1972), 257-263.

[8] T.P. Speed, "A note on commutative Baer rings III", J. Austral. Math. Soc. 15 (1973), 15-21.

Department of Mathematics,

University of Kansas,

Lawrence,

Kansas, USA. 